\title{
New addiction research unlikely to be applied
}

When scientists studying drug abuse meet on 9-10 August at the National Conference on Drug Abuse Prevention Research in Washington, DC, they will undoubtedly have much to discuss-including a widening gap between rapidly advancing basic science and applied research in the field.

In a flurry of recent studies, scientists have discovered that the mechanism of cocaine addiction is more complicated than previously thought, simultaneously revealing new molecular targets for drug addiction treatment and suggesting that multiple approaches will have to be combined to produce successful therapies. Despite the new findings, major pharmaceutical companies appear to show little interest in pursuing drug addiction treatments, raising questions about whether the promising new data can be translated into practical treatment.

A partial explanation for the power of addiction comes from recent studies using functional magnetic resonance imaging to map brain responses to monetary loss and reward. An examination of brain activity in 12 men playing a game of chance showed increased activity in the sublenticular extended amygdala, the nucleus acumbens and the hypothalamus when the volunteers anticipated winning money, mimicking the normal response to drugs like cocaine. The results indicate that addictive drugs target the processing of information about reward and loss-a powerful mechanism believed to be responsible for most human behavior (Neuron 30, 619; 2001).

Besides providing a strong neurochemical reward, cocaine also seems to usurp normal learning and memory processes, further inspiring users to seek the drug. Recent studies on rats yielded the surprising result that stimulating the dopaminerich medial forebrain bundle, the brain region responsible for cocaine's 'high', does not cause previously cocaine-addicted rats to resume self-administration of the drug (Science 292, 1178; 2001). In contrast, stimulating the glutamate-rich ventral subiculum of the hippocampus strongly motivates the rats to seek the drug again. The signal from the ventral subiculum requires glutamate in the ventral tegmental area, indicating that glutamate might be a useful target for drugs to treat cocaine addiction.

As the hippocampus is involved in con-

textual learning, the response of the rats seems to represent an association between the cocaine high and the context of the earlier cocaine experience. The phenomenon is similar to the behavior of human addicts who kick the habit, but then return to using cocaine when they encounter 'triggers' that cause them to remember previous highs. The relapse behavior of the rats indicates that addiction involves two distinct processes: the initial changes in neural circuitry induced by drug consumption, and the establishment of contextual memory traces in the hippocampus.

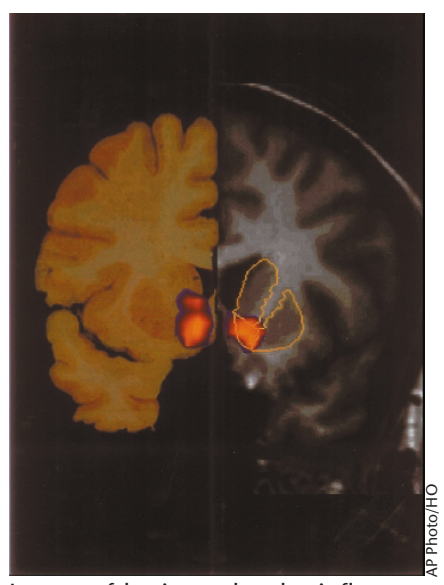

Image of brain under the influence of cocaine

the new findings on cocaine's action will lead to improved treatments for addiction.

In addition to new drugs targeting neurochemical pathways, addicts might eventually be able to turn to vaccines for help. At least two groups have developed active anticocaine vaccines that induce antibodies against the drug, and preclinical findings are promising. But Kathleen Kantak, who works on cocaine vaccines in the Department of Psychology at Boston University, warns, "It was never intended ... that the vaccine would be a stand-alone treatment for addiction. It would probably have to be used with other pharmacotherapies and counseling and behavioral techniques,"-primarily because the vaccine

Those memory traces may be quite easy to establish. Another team reports that a single exposure to cocaine induced longterm potentiation in the ventral tegmental area of mice for at least five days (Nature 411, 583; 2001). Preliminary results indicate that a similar mechanism might be involved in nicotine and morphine addiction.

Besides targeting glutamate to prevent relapse, future treatments for cocaine addiction might involve both the dopamine and serotonin transporters. Although dopamine metabolism has been a major focus of research on cocaine metabolism, researchers sponsored by the National Institute on Drug Abuse (NIDA) have found that dopamine and serotonin transporters are redundant in cocaine reward behavior (PNAS 98, 5300; 2001). Using knockout mice, the scientists found that the reward response to cocaine was inhibited only in animals lacking one or both copies of the serotonin transporter gene as well as both copies of the dopamine transporter gene.

Senior author on the NIDA paper, George Uhl, says the findings go "against generations of pharmacology where people tried to focus on a single site of action and target that with therapeutics." Previous efforts to treat cocaine addiction have generally targeted the dopamine transporter alone, and Uhl is hopeful that would not remove an addict's craving for the drug.

Despite the promising preclinical leads, major pharmaceutical companies have remained largely apathetic. Kim Janda, who has directed research on several cocaine vaccines at the Department of Chemistry at the Scripps Research Institute in La Jolla, told Nature Medicine that his team's work is funded primarily by a single biotechnology company, since neither NIDA nor the pharmaceutical industry has expressed much interest in developing cocaine vaccines.

Indeed, California-based Drug Abuse Sciences, which is funding Janda's work, appears to be the only company pursuing drug addicts as its principal market. Elizabeth Greetham, the company's CEO, thinks that big companies have avoided addiction treatment because of a "perception that addiction is a character flaw, and therefore it's psychosocial." However, according to Greetham's figures, $80 \%$ of alcoholics and $70 \%$ of cocaine addicts in the United States are employed, making that country's 6 million cocaine addicts and 10-12 million alcoholics a potentially lucrative market. The lead cocaine addiction treatment of Drug Abuse Sciences, a dopamine D1-receptor agonist, is now in Phase II clinical trials.

Alan Dove, Philadelphia 